\title{
Detection of a classical $\delta$ Scuti star in the new eclipsing binary system HIP 7666
}

\author{
E. Escolà-Sirisi ${ }^{1}$, J. Juan-Samsó ${ }^{1}$, J. Vidal-Sáinz ${ }^{1}$, P. Lampens ${ }^{2}$, \\ E. García-Melendo ${ }^{3}$, J. M. Gómez-Forrellad ${ }^{1,3}$, and P. Wils ${ }^{4}$ \\ ${ }^{1}$ Grup d'Estudis Astronòmics, Apdo. 9481, 08080 Barcelona, Spain \\ e-mail: duranobs@astrogea.org \\ 2 Koninklijke Sterrenwacht van België, Ringlaan 3, 1180 Brussel, Belgium \\ e-mail: patricia.lampens@oma.be \\ 3 Esteve Duran Observatory Foundation, Montseny 46 - Urb. El Montanya, 08553 Seva, Spain \\ e-mail: duranobs@astrogea.org \\ 4 Vereniging Voor Sterrenkunde, Belgium \\ e-mail: patrick.wils@cronos . be
}

Received 22 October 2003 / Accepted 15 December 2004

\begin{abstract}
HIP 7666 is a variable star newly discovered during the Hipparcos mission and classified as of unknown type (ESA 1997, The Hipparcos and Tycho Catalogues, ESA SP-1200). During 23 nights between July 2000 and November 2000, over 2300 CCD observations in the $V$ band were obtained. These data show that the new variable is a detached eclipsing binary system with an orbital period of 2.37229 days. In addition, one of the components undergoes very short-period oscillations with a main pulsation frequency of 24.46 or $25.47 \mathrm{c} / \mathrm{d}$. HIP 7666 is therefore a new member of the rare group of detached eclipsing binary systems with a $\delta$ Scuti type component.
\end{abstract}

Key words. stars: binaries: eclipsing - stars: oscillations - stars: variables: $\delta$ Sct - stars: individual: HIP 7666

\section{Introduction}

HIP 7666 (=HD 232486 = GSC 3671-1094 = NSV 15349) is a new variable star discovered by the Hipparcos mission (ESA 1997). No variable type could be assigned to this object and it was finally classified as unknown variable (flag “ $U$ ”). Aside from a determination of its $V$ magnitude, $(B-V)$ and $(U-B)$ color indices in a series of observations of stars with known accurate positions by Oja (1985), there appears to be no further investigation of this star in the literature. HIP 7666 was included in the Grup d'Estudis Astronòmics observing program (GEA 1999) after the discovery of its eclipsing binary nature in a search for new variable stars in this field. Subsequent observations showed short-period oscillations superimposed on the eclipsing binary light curve, indicating that at least one of the components is a possible $\delta$ Scuti star. Since very few eclipsing binary stars with pulsating components of this type are known, we decided to perform follow-up observations to verify the nature of the observed light variations and to identify one or more pulsation periods.

Eclipsing binaries are a source of accurate information on the physical properties of the components such as stellar radii and masses. If one of the components is in addition a (radial and/or non-radial) pulsating variable star, the system is of great scientific interest. The study of (non-radial) pulsators in such systems is very attractive for several more reasons:

- the near equator-on view of the stellar surfaces is a favourable configuration for the detection of sectoral modes (i.e. non-radial modes with $l=|m|$ );

- during the eclipses, the components act as a spatial filter which causes phase and amplitude modulation of the nonradial mode and makes the mode identification more accurate (Mkrtichian et al. 2002);

- the pulsating component can be correctly identified if there is evidence that the pulsations are most clearly present during one of both minima. Rodríguez et al. (2004) recently summarized the status of the eclipsing binaries with a $\delta$ Scuti component: only about a dozen cases are presently known. About $30 \%$ consist of eclipsing binaries with a classical $\delta$ Scuti pulsator while the rest defines a distinct class of $\delta$ Scuti pulsators, called the oscillating EA stars (Mkrtichian et al. 2002). The new data suggest that HIP 7666 is a normal main-sequence $\delta$ Scuti star in a detached eclipsing binary system. As such, the discovery of a $\delta$ Scuti pulsator in a "clean" binary system not affected by mass accretion and/or transfer is extremely important.

We present the newly collected photometric observations in Sect. 2. In Sect. 3, the photometric parameters are given and 
a Wilson-Devinney fit (hereafter WD, Wilson 1998) is computed to obtain a synthetic eclipsing binary light curve that can be subtracted from the data to analyse the residuals. The shortperiod light oscillations detected in the residual data are discussed in Sect. 4. In Sect. 5 we summarize the results of this work.

\section{Observations and reduction}

HIP 7666 was observed in the $V$ band from the Hostalets de Pierola Observatory ${ }^{1}$ using a $40 \mathrm{~cm}$ Newtonian telescope for 21 nights, and in the $B$ and $V$ bands from Monegrillo Observatory $^{2}$ also using a $40 \mathrm{~cm}$ Newtonian telescope for 9 and 4 nights respectively. All our observations were acquired between August and November 2000. The complete 34-night data set spans a time interval of 85 days. The observational $\log$ is listed in Table 1. Both telescopes, having identical optical systems, were equipped with a SX Starlight CCD camera with a Sony ICX027BL chip cooled by a Peltier system to about $-25{ }^{\circ} \mathrm{C}$. The field-of-view covered a sky region of $11.5^{\prime} \times 7.7^{\prime}$ and the pixel size was $1.80^{\prime \prime} \times 1.38^{\prime \prime}$. Dark frames and flat fields were obtained, and the standard image processing was carried out. The reduction was done using a software package called LAIA (Laboratory for Astronomical Image Analysis) and developed by Joan A. Cano ${ }^{3}$. Since the field of HIP 7666 is not crowded, the technique of aperture photometry was applied to extract the differential magnitudes.

The brightness of HIP 7666 was measured with respect to HIP 7665 (=HD 9976), while GSC 3671-1068 and GSC 3671-0834 served as check stars (Table 2). Although the comparison star is actually an optical pair (Dommanget \& Nys 1994) the photometric measurements were not affected by the component. In fact, thanks to the small angular separation between both components, and the faintness of the secondary component ( $\rho=1.1^{\prime \prime} ; \Delta_{H p}=2.65 \mathrm{mag}$, ESA 1997), HIP 7665 always appeared as a single object on all the CCD frames. The overall rms scatter of the differences between the comparison star and the principal check star is 8 mmag. Because no suitable check star could be included in the frames - other stars are at least 1 mag fainter than the comparison star -8 mmag is an upper limit to the true photometric error. Since the check star is 2.5 times fainter than the comparison star, we can infer that the rms scatter of the differences between the variable and the comparison star is about $5 \mathrm{mmag}$.

\section{The new binary system}

Figure 1 (upper panel) illustrates the light curves obtained in the $V$ and $B$ bands. The light curves are typical of an eclipsing binary system of the detached or semi-detached type. The observations reveal that HIP 7666 is an eclipsing binary system with a period of 2.37229 days which fades by 0.23 mag and 0.16 mag during, respectively, the primary and the secondary minimum. The total (peak-to-peak) amplitude in

\footnotetext{
${ }^{1}$ http://www. astrogea.org/jjs/index.html

2 http://www.astrogea.org/jvidal/index.html

${ }^{3}$ http://www.astrogea.org/soft/laia/laia.htm
}

Table 1. Observational log. In the fourth column HPO stands for Hostalets de Pierola Observatory and MO for Monegrillo Observatory.

\begin{tabular}{|c|c|c|c|}
\hline $\begin{array}{c}\text { Observation } \\
\text { date }\end{array}$ & $\begin{array}{c}\text { Observation interval } \\
\text { (HJD-2 } 451 \text { 000.0) }\end{array}$ & Filter & $\begin{array}{l}\text { Observatory } \\
\text { code }\end{array}$ \\
\hline 8 Aug. 2000 & $1765.553-1765.657$ & $V$ & HPO \\
\hline 9 Aug. 2000 & $1766.363-1766.664$ & $V$ & HPO \\
\hline 9 Aug. 2000 & 1766.376-1766.591 & $V$ & MO \\
\hline 10 Aug. 2000 & $1767.423-1767.586$ & V & HPO \\
\hline 10 Aug. 2000 & $1767.364-1767.590$ & $V$ & MO \\
\hline 12 Aug. 2000 & $1769.526-1769.667$ & $V$ & HPO \\
\hline 13 Aug. 2000 & $1770.407-1770.670$ & $V$ & HPO \\
\hline 14 Aug. 2000 & 1771.387-1771.672 & $V$ & HPO \\
\hline 15 Aug. 2000 & $1772.364-1772.644$ & $V$ & HPO \\
\hline 16 Aug. 2000 & $1773.384-1773.641$ & $V$ & HPO \\
\hline 17 Aug. 2000 & $1774.351-1774.664$ & $V$ & HPO \\
\hline 18 Aug. 2000 & $1775.397-1775.624$ & $B$ & MO \\
\hline 18 Aug. 2000 & $1775.365-1775.660$ & $V$ & HPO \\
\hline 19 Aug. 2000 & $1776.388-1776.604$ & $B$ & MO \\
\hline 19 Aug. 2000 & $1776.409-1776.524$ & $V$ & HPO \\
\hline 20 Aug. 2000 & $1777.395-1777.620$ & $V$ & HPO \\
\hline 24 Aug. 2000 & $1781.375-1781.635$ & $V$ & HPO \\
\hline 25 Aug. 2000 & $1782.376-1782.635$ & $V$ & HPO \\
\hline 28 Aug. 2000 & $1785.350-1785.560$ & $B$ & MO \\
\hline 31 Aug. 2000 & $1788.326-1788.598$ & $B$ & MO \\
\hline 1 Sep. 2000 & $1789.334-1789.545$ & $B$ & MO \\
\hline 2 Sep. 2000 & $1790.327-1790.586$ & $B$ & MO \\
\hline 4 Sep. 2000 & $1792.323-1792.479$ & $B$ & MO \\
\hline 4 Sep. 2000 & $1792.355-1792.582$ & $V$ & HPO \\
\hline 5 Sep. 2000 & $1793.386-1793.522$ & $V$ & HPO \\
\hline 7 Sep. 2000 & $1795.464-1795.464$ & $V$ & HPO \\
\hline 8 Sep. 2000 & $1796.313-1796.587$ & $B$ & MO \\
\hline 8 Sep. 2000 & 1796.372-1796.515 & $V$ & HPO \\
\hline 9 Sep. 2000 & $1797.361-1797.553$ & V & HPO \\
\hline 10 Sep. 2000 & $1798.370-1798.565$ & $B$ & MO \\
\hline 10 Sep. 2000 & $1798.373-1798.490$ & V & HPO \\
\hline 27 Oct. 2000 & $1845.277-1845.496$ & $V$ & MO \\
\hline 31 Oct. 2000 & $1849.281-1849.439$ & $V$ & MO \\
\hline 31 Oct. 2000 & $1849.373-1849.656$ & $V$ & HPO \\
\hline
\end{tabular}

Table 2. Catalogue data for the observed stars (ESA 1997).

\begin{tabular}{ccccc}
\hline \hline Star & Identifier & Sp. T. & $V_{T}$ & $(B-V)_{T}$ \\
\hline Var & HIP 7666 & A5 & 9.688 & 0.458 \\
Comp & HIP 7665 & A2 & 8.997 & 0.103 \\
Check1 & GSC 3671-1068 & G5 & 10.748 & 0.603 \\
Check2 & GSC 3671-0834 & - & 11.442 & 0.553 \\
\hline
\end{tabular}

$V$-light, including the ellipticity and reflection effects (causing the sinusoidal-like variations out-of-eclipse) in the close binary, is $0.25 \mathrm{mag}$. The measurements allowed us to compute a list of observed times of minima, presented in Table 3, from which we derived the following ephemeris by means of a linear least squares fit:

Min. I = HJD $2451769.5615(11)+2.37229(8) \times E$

where $E$ is the cycle number. Table 3 also lists the $(\mathrm{O}-\mathrm{C})$ residuals.

We made use of the WD code to model binary light curves (Wilson 1998) to obtain a preliminary photometric solution for 




Fig. 1. Upper panel: light-curve of HIP 7666 folded with the $2 \mathrm{~d} 37229$ period superimposed on the WD solution (upper curve: $\Delta V$, lower curve: $\Delta B$ ). Lower panel: residuals after subtracting the WD fit from the original photometric data (upper curve: $\Delta V$, lower curve: $\Delta B)$.

Table 3. Observed heliocentric times of minima.

\begin{tabular}{ccr}
\hline \hline HJD-2 450 000 & Type & O-C \\
\hline 1769.5620 & I & 0.0005 \\
1775.4888 & II & -0.0034 \\
1775.4946 & II & 0.0025 \\
1781.4203 & I & -0.0026 \\
1782.6166 & II & 0.0075 \\
1788.5385 & I & -0.0013 \\
1845.4748 & I & -0.0001 \\
1845.4750 & I & 0.0002 \\
\hline
\end{tabular}

the new binary. The available $V$ - and $B$-curves were simultaneously modelled in the case of a detached system (mode 2). A semi-detached solution was also tested, but the best fits resulted with residuals an order of magnitude worse than for a detached configuration. We adopted a surface temperature of $8200 \mathrm{~K}$ (Schmidt-Kaler 1982) for the primary star corresponding to the star's global spectral type of A5 (Cannon \& Pickering 1918-1924). Other assumptions were: a radiative bolometric albedo $\left(A_{1}=1\right)$ and gravity darkening $\left(g_{1}=1\right)$ for the primary, a convective bolometric albedo $\left(A_{2}=0.5\right)$ and gravity darkening $\left(g_{2}=0.32\right)$ for the secondary, and a logarithmic limb darkening law (Van Hamme 1993). We used the known spectral type to estimate the surface temperature because the wide-band photometry (Oja 1985) does not allow us to compute the reddening, which could be significant given its low Galactic latitude $\left(-9.7^{\circ}\right)$. Indeed, making use of the Strömgren measurements of the hotter star HIP 7729 (=HD 10054) (Westin 1982), which, with a parallax of $\approx 3$ mas (ESA 1997), is at about the
Table 4. Parameters of the binary models which gave equivalent best fits.

\begin{tabular}{cccc}
\hline \hline$T_{1}(\mathrm{~K})$ & $T_{2}(\mathrm{~K})$ & Sp. Type 2 & Range for $i$ \\
\hline 9000 & 7300 & A9-F0 & $82.0-79.0$ \\
8200 & 6700 & F3-F4 & $81.0-78.5$ \\
7500 & 6200 & F7-F9 & $81.0-78.5$ \\
\hline
\end{tabular}

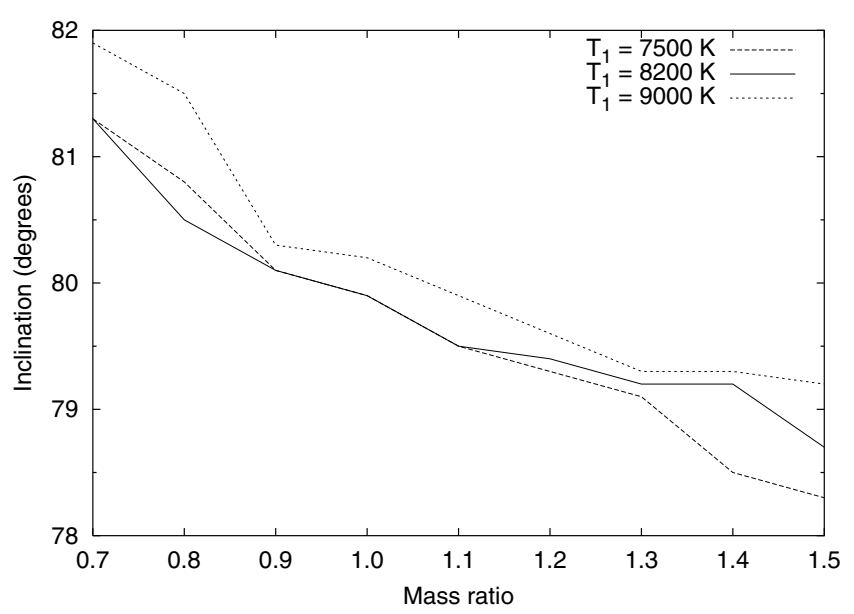

Fig. 2. The inclination as a function of the mass ratio for three different values of the primary's surface temperature.

same distance as HIP 7666 and in almost the same direction, we obtain a colour excess $E(b-y)$ of 0.1 mag after application of the calibration procedure of the Strömgren photometric system (Moon \& Dworetsky 1985). Because of the large uncertainty on the dereddened colour indices, we preferentially made use of the spectral type classification.

Since no radial velocity information is available, we searched for the best solution by fixing the mass ratio $q=$ $M_{2} / M_{1}$ and exploring a large number of values between 0.5 and 4.0. The surface temperature of the secondary, $T_{2}$, the gravitational potential for both components, the relative monochromatic luminosities $L_{1}$ and $L_{2}$, and the orbital inclination $i$ were considered to be free parameters. Since the eclipses are partial, and given the observational uncertainties, it was not possible to derive the photometric mass ratio in a reliable way and all $q$-values between 0.7 and 1.5 gave equivalent fits. However, the surface temperature of the secondary, $T_{2}$, does not depend much on the choice of $q$ within the given range. Table 4 lists the parameters of the best fit when $T_{1}$ equals $8200 \mathrm{~K}$. We next investigated the parameter space using different values for the primary's surface temperature assuming an uncertainty of half a spectral class: adopting $T_{1}=9000 \mathrm{~K}$ (A0-type star) or $T_{1}=7500$ (F0-type star) however did not alter the quality of the fit significantly. In these cases, the secondary is $\approx 1500 \mathrm{~K}$ cooler than the primary (see Table 4). In Fig. 2 the value of the inclination is represented as a function of the mass ratio $q$ for three values of the surface temperature $T_{1}$. Note that a mean value of $80 \pm 2^{\circ}$ reflects well the range of possible values.

Though modelling of the $B$ - and $V$-curves did not allow us to constrain the physical parameters of the components 
uniquely or accurately, one can see that the match between one of these best solutions (we chose $q=0.9$ ) and the observed light curves is very good (Fig. 1, upper panel). This is especially obvious in the plot of the residuals where the peak-topeak differences are of the order of $40 \mathrm{mmag}$ at most (Fig. 1, lower panel).

\section{Detection of short-period oscillations}

In the plot of the residual light curves we notice periodic fluctuations with a maximum difference of $20 \mathrm{mmag}$. Despite their small amplitude, these are real as we could detect them during two different nights of simultaneous observations at Hostalets de Pierola Observatory and Monegrillo Observatory in Spain. Both observatories are about $300 \mathrm{~km}$ apart and are equipped with similar instrumentation (cf. Sect. 2). To rule out the possibility of observing variations caused by the comparison star, independent photometry of HIP 7666 and HIP 7665 was performed with respect to the check stars GSC 3671-1068 and GSC 3671-0834 (cf. Table 2). Figure 3 illustrates the photometric runs taken from Hostalets de Pierola Observatory and Monegrillo Observatory on the night corresponding to JD 2451766.5. The new photometric data confirmed that HIP 7666 exhibits small-amplitude variations whereas the comparison star does not. Figure 4 shows the brightness variations for that particular night, after merging and averaging the data from both observatories. It can be seen that the maximum amplitude variation is about $20 \mathrm{mmag}$. The amplitude modulation present in this light-curve segment further suggests the presence of more than one frequency. The existence of short-period oscillations superimposed on the (eclipsing) binary light-curve is clearly demonstrated. Because of the short periodicity and the amplitude modulation, pulsations in one (or both) of the components are presumably the cause of these rapid light variations.

\subsection{The data during the phases of eclipse}

From the $V$-light curve in Fig. 1 it seems that the residuals at the phase of primary minimum are somewhat larger than those of secondary minimum. No conclusion can be formulated from the incomplete $B$-light curve. Due to the mentioned above uncertainties, however, we have no direct clue as to which component may be the pulsating star, but it may be supposed that the primary star which contributes most to the light is a good candidate. On the basis of the broad range in the spectral classification alone, both components may lie in the $\delta$ Scuti instability strip. The primary could be a main sequence pulsator, while the secondary (which is cooler) could be a more evolved $\delta$ Scuti star.

\subsection{The data out-of-eclipse}

In order to perform a Fourier analysis in the frequency domain, we removed the WD fit from the original data set. First only the data out-of-eclipse were analysed using Period98 (Sperl 1998). The power spectrum is dominated by a lot of noise at frequencies smaller than $10 \mathrm{c} / \mathrm{d}$, probably due to a combination

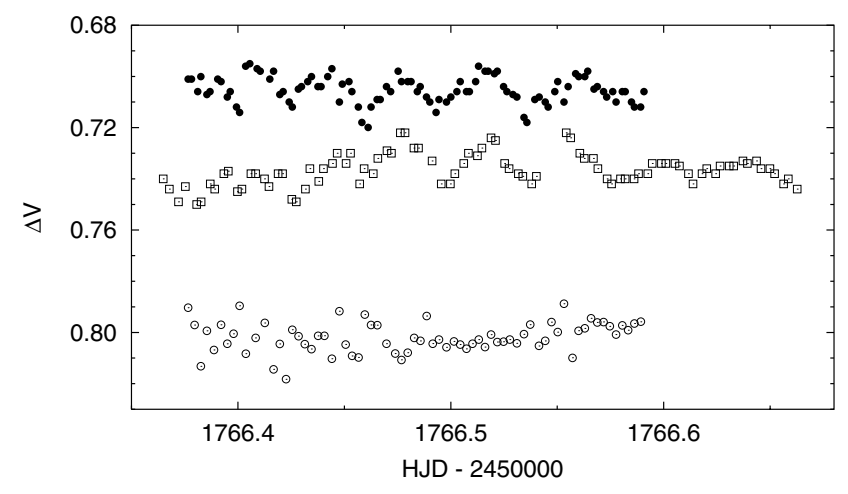

Fig. 3. Comparison between the light curves of HIP 7666 taken from Monegrillo Observatory (filled dots, upper curve), and Hostalets de Pierola Observatory (open squares, middle curve). Both data series show the same light variations which are not present in the data of the comparison star with respect to the two check stars GSC 36711068 and GSC 3671-0834 collected at Monegrillo Observatory (open circles, bottom curve, note that these were shifted by a constant magnitude offset to fit in the plot). Note that since the check star is much fainter, the scatter is correspondingly higher.



Fig. 4. Light curve of HIP 7666 taken on JD 2451766.5 obtained after merging and averaging the data from Monegrillo and Hostalets de Pierola Observatories.

of nightly zero-point shifts and other observational errors. This situation did not change when the residual data during eclipse were included. In the less noisy part of the periodogram (in the range $>10 \mathrm{c} / \mathrm{d}$ ) the highest peaks correspond to $24.463 \pm$ $0.008 \mathrm{c} / \mathrm{d}$ and its one day ${ }^{-1}$ alias at $25.465 \pm 0.008 \mathrm{c} / \mathrm{d}$ with a semi-amplitude of $1.7 \mathrm{mmag}$, introduced by the daily gaps in the observing pattern (see Fig. 5). The quoted error bar corresponds to the half-width at half maximum of the peaks in the power spectrum. This is truly an upper limit of the error (Schwarzenberg-Czerny 1991). The $S / N$ ratio for this frequency is 4.1 and is just significant. This is illustrated by Fig. 6 .

Figure 7 shows the power spectrum of the residual data after removing the $\delta$ Scuti pulsations, where the spectrum is noisy with most of the energy concentrated at frequencies below $10 \mathrm{c} / \mathrm{d}$. Figure 8 displays the folded light curve. Although the light curve of Fig. 4 presents a vague hint of multiperiodicity, no other reliable frequency could be found in that range of frequencies larger than $>10 \mathrm{c} / \mathrm{d}$, as expected from the explicited observational error and the low $S / N$ ratio. 


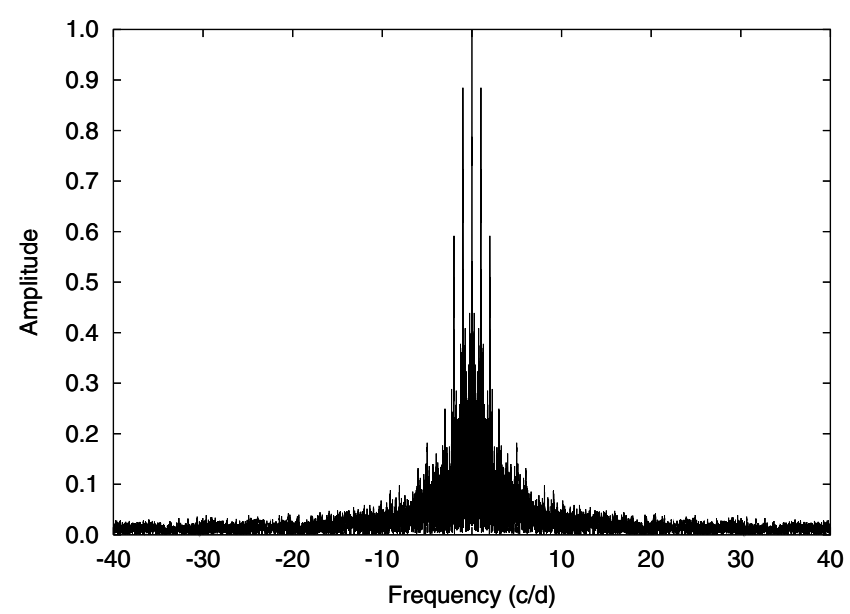

Fig. 5. Spectral window for the observations of HIP 7666.



Fig. 6. Power spectrum of the prewhitened out-of-eclipse data where the $24.46 \mathrm{c} / \mathrm{d}$ component is marked as $\mathbf{f}$.

\section{Discussion}

Given the very short periodicity of about $1 \mathrm{~h}$ and the location in the $H-R$ diagram (a star of spectral type A5V lies in the region where the classical instability strip intersects the mainsequence), we conclude that at least one of the components of HIP 7666 is a pulsating star of type $\delta$ Scuti. Analysis of the light curve of the eclipsing system further suggests that one component is lying in the hotter part of the $\delta$ Scuti instability strip while the other component may just lie outside it, being probably cooler than its red border (cf. Breger 2000). We therefore propose that the primary component is a $\delta$ Scuti pulsator.

There are only a very few known normal $\delta$ Scuti stars that are members of detached eclipsing binary systems (see Rodríguez 2002; Rodríguez et al. 2004). Recent investigations have demonstrated the existence of a group of A-F pulsating stars located in the $\delta$ Scuti instability strip and with the same observational characteristics as $\delta$ Scuti stars but in a different evolutionary stage. This new group consists of mass-accreting main-sequence pulsating components of semi-detached Algol-type binaries (Mkrtichian et al. 2002). Presently thirteen members have been discovered, including

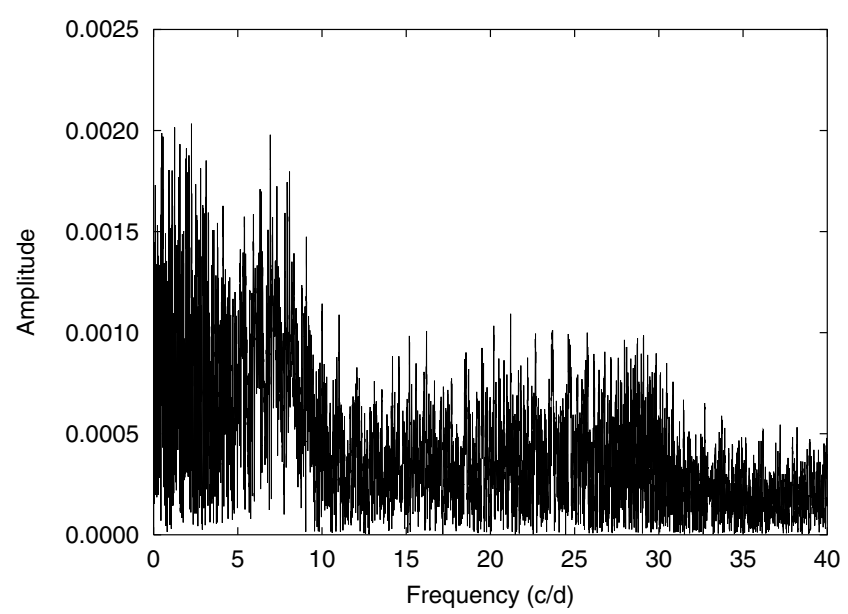

Fig. 7. Power spectrum of the residuals data after prewhitening the $\delta$ Scuti pulsation signal.

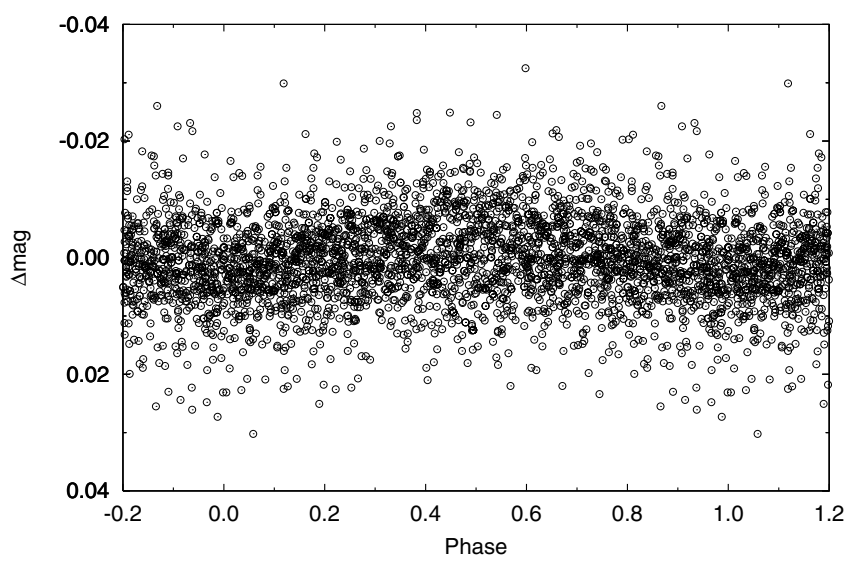

Fig. 8. Folded light-curve of the prewhitened out-of-eclipse data folded on the 0.981 hour-period.

the latest discoveries (Caton 2004; Kim et al. 2004a,b; Lampens et al. 2004), but excluding WX Eri (Arentoft et al. 2004). They have orbital periods ranging from 1 to 7 days and pulsation periods from $22 \mathrm{~min}$ to $6.5 \mathrm{~h}$. One complex example is RZ Cas (Mkrtichian et al. 2003).

Our observations suggest that HIP 7666 is a classical $\delta$ Scuti star in a detached eclipsing binary system, which probably does not belong to the previous class. Nevertheless, the interest of such a discovery is high since these objects allow one to study the undisturbed pulsation characteristics while also providing the basic stellar properties needed to better constrain the pulsation models. Further observations are necessary, in particular high-resolution spectroscopic data would be helpful to confirm the precise nature of the binary system and to fully explore the nature of the detected pulsations. We would welcome new collaborations in this respect, as we have already started an extensive, multi-site photometric campaign.

Acknowledgements. The authors are pleased to acknowledge Prof. R. Wilson (University of Florida) for the Wilson-Devinney code. P. Lampens acknowledges support from the Fund for Scientific Research (FWO) - Flanders (Belgium) (project G.0178.02). We further thank 
Joan A. Cano and Rafael Barberá for writing the software for obtaining and reducing the $\mathrm{CCD}$ frames. We are also greateful to the anonymous referee whose comments helped to improve this paper. We made use of the Simbad database, operated by the Centre de Données astronomiques de Strasbourg (France), and the ADS service.

\section{References}

Arentoft, T., Lampens, P., Van Cauteren, P., et al. 2004, A\&A, 418, 249

Breger, M. 2000, in Delta Scuti and Related Stars: Reference Handbook, ed. M. Breger, \& M.H. Montgomery, ASP Conf. Ser., 210,6

Cannon, A. J., \& Pickering, E. C. 1918-1924, Henry Draper Catalogue and Extension 1 (HD, HDE), Harv. Ann. 91-100

Caton, D. B. 2004, IBVS, No. 5531, 1

Cuypers, J. 1987, Medelingen van de Koninklijke Academie voor Wetenschappen, Letteren en Schone Kunsten van België, Jg. 49, Nr. 3, ed. Paleis der Academiën, Brussel, 21

Dommanget, J., \& Nys, O. 1994, Commun. Obs. Roy. Belg., 115, 1

ESA 1997, The Hipparcos and Tycho Catalogues, ESA SP-1200

Grup d'Estudis Astronòmics, GEA 1999, http://www . astrogea.org/instrumental/patrol.htm

Kim, S.-L., Koo, J.-R., Lee, J.A., et al. 2004, IBVS 5537, No. 1

Kim, S.-L., Kang, Y. B., Koo, J.-R., Mkrtichian, D. E., \& Lee, J. W. 2004, IBVS 5538, No. 1

Lampens, P., Van Cauteren, P., Strigachev, A., et al. 2004, IBVS, No. 5572,1
Mkrtichian, D. E., Kusakin, A. V., Gamarova, A. Yu., \& Nazarenko, V. 2002, ASP Conf. Ser., 259, 96

Mkrtichian, D. E., Nazarenko, V., Gamarova, A. Yu., et al. 2003, in Interplay of periodic, cyclic and stochastic variability in selected areas of the H-R diagram, ed. C. Sterken, ASP Conf. Ser., 292, 113

Montgomery, M., \& O’Donoghue, D. 1999, Delta Scuti Newsletter, 13,28

Moon, T. T., \& Dworetsky, M. M. 1985, MNRAS, 217, 305

Oja, T. 1985, A\&AS 59, 461

Rodríguez, E. 2002, in Proceedings of the First Eddington Workshop on Stellar Structure and Habitable Planet Finding, 11-15 June 2001, Córdoba, Spain, ed. F. Favata, I. W. Roxburgh, \& D. Galadi, ESA SP-485, 323

Rodríguez, E., García, J. M., Gamarova, A. Y., et al. 2004, MNRAS, 347, 1317

Schmidt-Kaler, T. H. 1982, Physical parameters of stars, in LandoltBörnstein New Series, Vol. 2b, ed. K. Schaifers, H.H. Voigt (NewYork: Springer-Verlag)

Schwarzenberg-Czerny, A. 1991, MNRAS, 253, 198

Sperl, M. 1998, Comm. in Asteroseismology, 111, ed. Breger, M. (Vienna), 1

Wilson, R. E., Computing Binary Star Observables, (Reference manual to the Wilson-Devinney program), Department of Astronomy, University of Florida, Gainesville, FL, 1998 edition

Van Hamme, W. 1993, AJ, 106, 2096

Westin, T. N. G. 1982, A\&AS, 49, 561 